\title{
Recent breakthroughs in hollow core fiber technology
}

Gregory Jasion, Thomas Bradley, Hesham Sakr, John Hayes, Yong Chen, et al.

Gregory T. Jasion, Thomas Bradley, Hesham Sakr, John R. Hayes, Yong Chen, Austin Taranta, Hans Christian Mulvad, Ian A. Davidson, Natalie V. Wheeler, Eric Numkam Fokoua, Wei Wang, David J. Richardson, Francesco Poletti, "Recent breakthroughs in hollow core fiber technology," Proc. SPIE 11309, Next-Generation Optical Communication: Components, Sub-Systems, and Systems IX, 1130902 (31 January 2020); doi: 10.1117/12.2548585

SPIE. Event: SPIE OPTO, 2020, San Francisco, California, United States 


\title{
Recent Breakthroughs in Hollow Core Fiber Technology
}

\author{
Gregory T. Jasion, Thomas Bradley, Hesham Sakr, John R. Hayes, Yong Chen, Austin Taranta, \\ Hans Christian Mulvad, Ian A. Davidson, Natalie V. Wheeler, Eric Numkam Fokoua, Wei Wang, \\ David J. Richardson and Francesco Poletti \\ Optoelectronics Research Centre, University of Southampton, SO17 1BJ, UK
}

\begin{abstract}
Flexible dielectric optical fibers guiding light in a hollow core were conceptually imagined at the end of the $19^{\text {th }}$ century, but first demonstrated in practice about 2 decades ago. Since then, many geometric variants have been described and implemented, and theoretical models developed and finessed. Despite this, for a fairly long time the key metric by which their performance was judged - attenuation - has remained quite considerably higher than standard all-glass fibers. In this paper, we describe the recent breakthroughs in hollow core fiber technology. We trace the story of this breakthrough from the theoretical exploration of a new design of hollow core fiber, through early implementations, up to the staggering results achieved over the last 18 months. The progress reported concerns not only a reduction in the fiber attenuation level, but also a considerable improvement in modal quality of the fibers, which have led to excellent data transmission performance. These fabricated fibers tell a story of improvements in all aspects of the technology, including preform preparation, performance modelling, fiber draw dynamics and coatings.
\end{abstract}

Keywords: Hollow core fiber, NANF, TELECOMS

\section{INTRODUCTION}

Hollow core optical fibers (HCF), in which light is guided in an air or vacuum core, have attracted scientists for over a century due to their low non-linear response, low latency and lower dispersion as compared to solid fibers. Many of the limitations of conventional optical fiber originate from the fact that light propagates in solid glass. One might thus desire to replace glass with air or vacuum, but light is not guided effectively in a simple holey channel in a bulk glass filament. Instead, the hollow core must be surrounded by a microstructured dielectric cladding that somehow confines the light to the core. The first such cladding to be demonstrated in a fabricated fiber was presented in $1999^{1}$. Since this first venture, many advances in the fibers' optical properties have been demonstrated, making their use attractive to numerous applications ${ }^{2,3}$. However, for application in optical communications, by far the largest of the possible markets, the optical loss of the fibers was - and in some respect still is - too high. Before 2018, the lowest rigorously documented loss in an HCF was $1.7 \mathrm{~dB} / \mathrm{km}$ and was obtained in a photonic bandgap guiding HCF (PBGF) reported in 2004. More recently a new design for HCFs was proposed, where the hollow core is surrounded by a microstructure cladding composed of tubes attached to the jacket glass but leaving a small gap between each other. By avoiding contact between the tubes, the cladding is composed of only a single thickness, resulting in a wide smooth transmission window ${ }^{5}$. The simplicity of this structure and the broad wide bandwidth has made it extremely popular and several further examples were demonstrated achieving losses as low as $8 \mathrm{~dB} / \mathrm{km}$, which, while impressive, is still too high for most data communication applications ${ }^{6}$. Such a tubular hollow core design has an additional geometric benefit which, if leveraged effectively, can make higher order modes extremely high loss and render the fiber effectively single moded. By choosing the size of the capillaries in the cladding carefully, the modes guided within those capillaries can have similar propagation constants as higher order modes (HOMs) guided in the core. These air guided HOMs will then be stripped out of the core into the high loss cladding, leaving after some distance only the fundamental core mode. Such effectively single mode designs have been demonstrated ${ }^{6,7}$, for example Fig.1(a), and typically a cladding of 7 tubes is chosen which allows for small gaps, low fundamental mode loss and high HOM extinction.

\subsection{The Nested Anti-resonant Nodeless Fiber (NANF)}

A further development to the tubular design was then proposed, in which smaller tubes are nested inside the tubes that make up the cladding. These smaller inner tubes are also attached only to the jacket glass and their thickness is equal to that of the larger tubes ${ }^{8}$. The Nested Antiresonant Nodeless Fiber (NANF) described in this theoretical study demonstrated 
how the second anti-resonant element reduced the loss considerably and provided a route for hollow core fibers to be made with loss below that of telecom standard SMF.

The Nested Antiresonant Nodeless Fiber is a hollow core fiber that confines light using the anti-resonance principle. When the thickness of all the capillaries forming the cladding of the fiber is chosen to be in anti-resonance with the desired operational wavelength, this nodeless structure gives a smooth and wide transmission window. In general, for all HCFs there is always a contribution to the overall loss coming from confinement or leakage, since there is no total-internal reflection to guide light in the core. In a NANF the confinement loss is a function of the geometry and the key parameters are: the size of the core, the size of gaps between primary capillaries, and the size of the nested element. The nested element will also control the size of the cavities in the cladding to promote HOM extinction and single moded performance.

Anti-resonant fibers (ARFs) can confine light in the core over broadband spectral regions aside from where resonances with the membranes occur. The spectral position of these resonances can be calculated by a simple expression: $\lambda_{m}=$ $\frac{2 t}{m} \sqrt{n^{2}-1}, m=1,2,3 \ldots$, where $t$ is the thickness, $n$ is the refractive index of the glass and $m$ is an integer value indicating the order of the resonance. The resonances are evenly spaced in frequency and between them anti-resonant windows open in which the fiber transmits light with low loss. The first anti-resonance window is the widest (in wavelength domain) and is located at about 1.5x the wavelength of the first resonance. From this simple expression one can estimate that for an ARF to guide in the telecommunications C-band around $1550 \mathrm{~nm}$ in the $1^{\text {st }}$ or $2^{\text {nd }}$ window a membrane thickness of approximately $550 \mathrm{~nm}$ or $1200 \mathrm{~nm}$, respectively, would be needed. The first window has larger bandwidth, making it optically superior, and also provides a $2 \mathrm{x}$ higher fiber yield per perform; however, the thinner struts and the higher draw down ratio make it more challenging to fabricate since both factors increase their sensitivity to pressure ${ }^{9}$.

Several groups have reported excellent progress towards realizing this NANF geometry, however, none of the fibers yet reported have the required structure or symmetry to achieve the low loss values predicted ${ }^{10,11}$. In early 2018 a significant step towards low-loss ARF was reported using a different approach to produce a double membrane tubular-like fiber ${ }^{12}$, where glass sheets were inserted into the preform to divide the non-contact tubes into a conjoined pair. The conjoined fiber achieved a low loss of $2 \mathrm{~dB} / \mathrm{km}$ in a fiber over $300 \mathrm{~m}$ long, the lowest loss of any ARF at the time. The chosen design, however, formed glass nodes in the cladding, which introduced resonances into the transmission window and reduced the useable bandwidth.
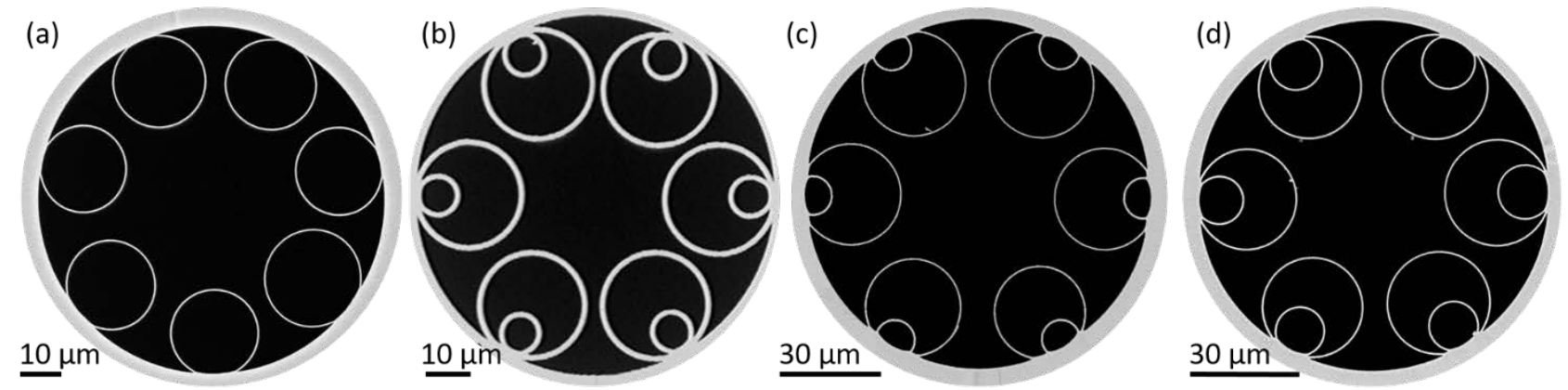

Figure 1. The progress of our hollow core anti-resonant fiber structures ordered chronologically, (a) Tubular fiber with large bandwidth $^{7}$, (b) the first low loss NANF ${ }^{13}$, (c) the first low loss NANF operating in the first anti-resonant window ${ }^{14}$, (d) the lowest loss hollow core fiber ever made ${ }^{15}$.

In this work, we describe the development of the first realized low loss NANF ${ }^{13}$, which became the lowest loss hollow core fiber of any kind, Fig. 1(b). We then report the subsequent development of a NANF with operation in the first antiresonant window ${ }^{14}$, giving it larger bandwidth and longer yield, Fig. 1(c), although with slightly compromised loss due to geometrical asymmetries. Finally, we describe the latest developments in this ongoing journey which have produced a data transmitting fiber operating in the first anti-resonant window and with losses $<1 \mathrm{~dB} / \mathrm{km}$, centered at $1550 \mathrm{~nm}$, and a length of over $1 \mathrm{~km}^{15}$, Fig. 1(d).

\section{THE FIRST LOW LOSS NANF}

In 2018 we reported the first low loss Nested Antiresonant Nodeless Fiber (NANF) achieving a broad smooth transmission window and a low loss of $1.3 \mathrm{~dB} / \mathrm{km}$ at $1450 \mathrm{~nm}$. The fiber was $0.5 \mathrm{~km}$ long, was demonstrated to be effectively single moded after a $30-50 \mathrm{~m}$ and able to transmit data with low penalty ${ }^{13}$. This first NANF structure was designed to have the 
second antiresonant window centered at 1550nm, requiring relatively thick tube membranes of 1150nm - which simplified the fabrication task. A structure with 6 outer elements with nested capillaries of a carefully designed size was chosen as a compromise between good HOM suppression and low loss for the fundamental mode.


Figure 2: (a) (Top) Transmission through long and short fiber lengths and average cutback loss, (Bottom) zoomed in cutback with measurement error (shaded area); (b) comparison between measure NANF loss and previous HCF loss record ${ }^{4}$; (c) Total simulated loss (black) vs measurement (red) and various loss contributions: confinement (blue dash), scattering (red dot); microbend (green, dash-dot).

The fiber was fabricated from the typical two-stage stack and draw method: the cladding capillaries were made with a similar aspect ratio as for the fiber design but about 200x larger. The first stage was fabricated by stacking and fusing capillaries in a slender jacket tube, this assembly was then drawn into canes, then jacketed and drawn to the final fiber. Distinct pressures were applied to the core, outer capillaries, and nested capillaries; the pressures were chosen with the assistance of fluid dynamics draw modelling used during the draw ${ }^{9}$.

The drawn fiber had a core size of $31 \mu \mathrm{m}$, the structure of this fiber can be seen in Fig. 1(b), it is extremely uniform with a range of tube thicknesses of the outer capillaries measured as $1170 \pm 29 \mathrm{~nm}$, the inner capillaries were slightly thinner than intended at $1080 \pm 25 \mathrm{~nm}$. The thickness mismatch of approximately $100 \mathrm{~nm}$, while small, is responsible for a small reduction in bandwidth and the slightly short position of the transmission window centered at $1450 \mathrm{~nm}$ rather than 1550 $\mathrm{nm}$. The fiber draw was very stable and controllable, and remarkably small inter-tube azimuthal gaps down to $\sim 1$ wavelength could be achieved and maintained along the full fiber length.

The fiber was then spooled on a 1-m circumference bobbin and cut back to $10 \mathrm{~m}$. Its spectral transmission curves and cutback loss are shown in Fig. 2(a). Spectral transmission measurements were acquired (for three different cleaves) at both long and short fiber lengths. The red solid line indicates the average loss and the grey shaded area around it the measurement error. The minimum loss region falls short of the C-band (something that can be straightforwardly addressed in future draws), and is centered around $1450 \mathrm{~nm}$. The fiber has a loss below $1.4 \mathrm{~dB} / \mathrm{km}$ over a bandwidth of $40 \mathrm{~nm},<1.5$ $\mathrm{dB} / \mathrm{km}$ over $65 \mathrm{~nm}$ and $<2 \mathrm{~dB} / \mathrm{km}$ over $90 \mathrm{~nm}$. Figure 2(b) shows how its second transmission window loss compares with the previous HCF record loss ${ }^{4}$.

The measurement is further supported by simulations, as shown in Fig. 2(c). Image processing was used to extract accurate structural information from SEMs, and mass conservation from the preform was imposed to improve accuracy in estimating individual tube thicknesses. Three loss contributions were evaluated: confinement from FEM calculations; surface 
scattering ${ }^{8}$, and microbend from the coupled power theory model ${ }^{16}$. As can be seen, by summing up all contributions, excellent agreement is observed not only for the $2^{\text {nd }}$ window around $1450 \mathrm{~nm}$, but also for the $3^{\text {rd }}$ window around $950 \mathrm{~nm}$. At $950 \mathrm{~nm}$ the minimum loss of $1.8 \mathrm{~dB} / \mathrm{km}$ is also a record value and only $2 \mathrm{x}$ higher than Rayleigh scattering in silica. The observation that confinement and microbend dominate the loss in the $2^{\text {nd }}$ and $3^{\text {rd }}$ window respectively indicate potential for improvements through optimizing the microstructure geometry and the coating package.

To assess the modal behavior of the fiber, a $10 \mathrm{~m}$ and $495 \mathrm{~m}$ length were tested using an $\mathrm{S}^{2}$ system. A clear $\mathrm{LP}_{11}$ peak is present after 10 meters, but this becomes attenuated below the measurement noise floss in the long length. An experimental S2 cutback indicates losses of LP11, LP21 and LP02 modes as high as 640,750 and $2100 \mathrm{~dB} / \mathrm{km}$ respectively. To test the data transmission potential of this fiber it was spliced to SMF tails and connected to a $10 \mathrm{G} \mathrm{SPF}+80 \mathrm{~km}$ reach transceiver, with a total insertion loss of $3.5 \mathrm{~dB}$ at $1550 \mathrm{~nm}$. A Bit Error Ratio (BER) of $10^{-9}$ was achieved for a received power of $-24.6 \mathrm{dBm}$. The fiber was then left under test for over 60 hours at a received power of $-20 \mathrm{dBm}$ and no errors were detected. This indicates the absence of an error floor at the $10^{-15} \mathrm{BER}$ level and confirms the expected low levels of intermodal coupling and multi-path interference.

\section{MOVING TO THE $1^{\text {ST }}$ ANTI-RESONANCE WINDOW}

The excellent optical performance of the fiber described in the previous section can be greatly improved by making one simple change: operating in the $1^{\text {st }}$ anti-resonance window. Figure 3 compares the confinement loss of the $2^{\text {nd }}$ window NANF geometry, extracted from SEM images, and the same structure modified to have an average thickness of $550 \mathrm{~nm}$ to center the first anti-resonance window in the telecoms C-band. The minimum loss of both structures is comparable, however, the bandwidth at $2 \mathrm{~dB} / \mathrm{km}$ of the $1^{\text {st }}$ window is $\sim 3 \mathrm{x}$ larger than for $2^{\text {nd }}$ window operation, making a $1^{\text {st }}$ window structure clearly preferable.

Targeting a first window geometry is more difficult, since during the draw the thinner capillary walls offer less viscous stress and so are more sensitive to changes from pressure and surface tension. This change in draw dynamics brings the mid-draw contact threshold closer to the target geometry, limiting the minimum size of the gaps that can be achieved in practice $^{9}$. However, achieving a structure with high optical qualities is still possible, and a fiber with several $100 \mathrm{~nm}$ of bandwidth spanning the telecoms bands with low flat dispersion and near vacuum latency is a worthy pursuit.

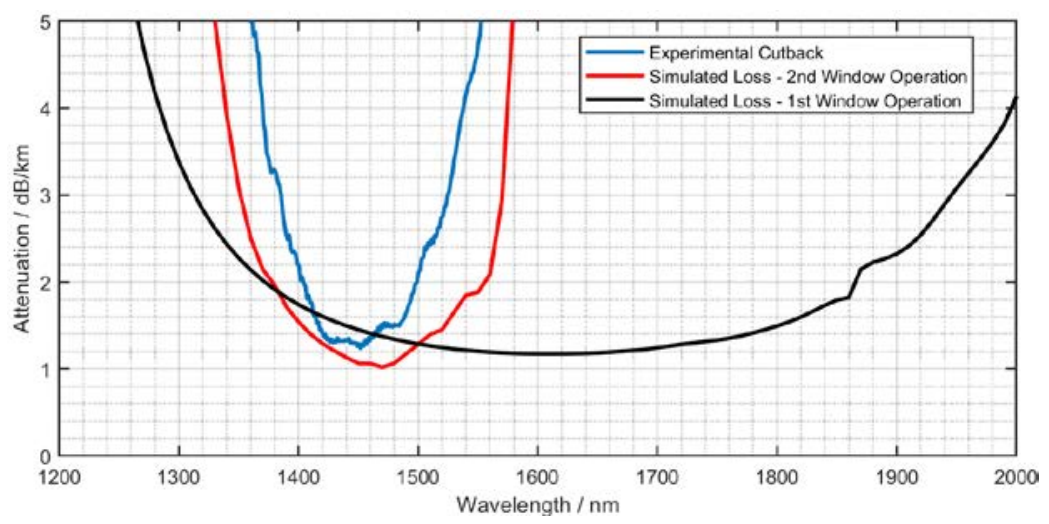

Figure 3. Experimental cutback (blue) and a simulation of the experimental geometry (red) both operating in the $2^{\text {nd }}$ antiresonance window. Compared to a simulation result showing the much larger bandwidth of the $1^{\text {st }}$ anti-resonance window (black), thickness has been changed but the geometry is otherwise the same.

At the beginning of 2019 we reported the first ultrawide band low loss NANF operating in the first anti-resonance window $^{14}$. The targeted structure had a core of $35 \mu \mathrm{m}, 6-8 \mu \mathrm{m}$ gaps and small nested elements to promote HOM extinction. This realized fiber presented some asymmetries in its cross-section, namely some tube size and thickness variation, and as a result its loss was $6.6 \mathrm{~dB} / \mathrm{km}$, as measured by cutback, Fig. 4(a). Using simulation tools, we were able to decompose the loss into confinement loss, scattering and microbend, and achieve good overall agreement with the measured loss, as before. Surface scattering contributions to the overall loss remains small, but confinement loss contributes about two thirds of the loss at $1550 \mathrm{~nm}$, this is due to the large gaps and structural asymmetry. Using simulation data, we quantified the azimuthal variation of the leakage loss contribution around the edge of the microstructure perimeter. We identified that the larger gaps on either side of a capillary which was smaller than the rest were already responsible for $\sim 1.5 \mathrm{~dB} / \mathrm{km}$ of confinement loss, Fig. 4(c). Figure 4(d) compare this first window fiber with the fiber reported above. Our 
simulations at that time also suggested that further improvements in uniformity could reduce the loss to less than $4 \mathrm{~dB} / \mathrm{km}$, and with improvements to pressurization we could reduce the gap size and the losses would be further reduced. Finally, countering the effects of microbend would enable us to achieve a loss below $1 \mathrm{~dB} / \mathrm{km}$. The modal qualities of this fiber were tested using $\mathrm{S}^{2}$ method on $10 \mathrm{~m}$ and $351 \mathrm{~m}$ lengths, Fig. 4(b). In $10 \mathrm{~m}$ the $\mathrm{LP}_{11}$ mode is clearly visible, but after 350 $\mathrm{m}$ all the HOMs have been heavily attenuated and the fiber is effectively single mode.
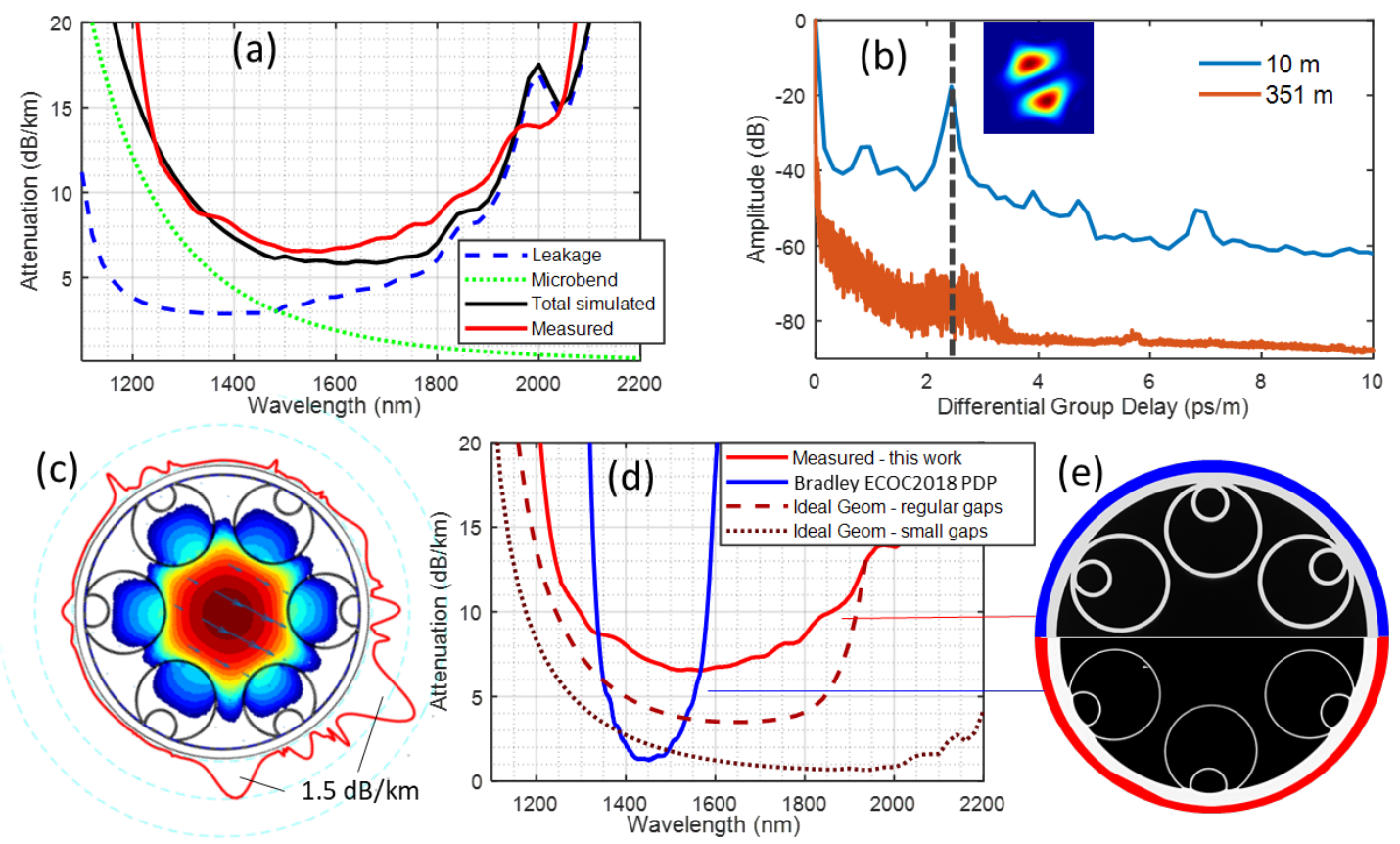

Figure 4. (a) Measured versus simulated loss; (b) $\mathrm{S}^{2}$ measurement showing that no appreciable high order mode content remains after 350m; (c) simulated fundamental mode and its radial Poynting vector, showing that a considerable fraction of the loss arises from the gaps created by a small tube; (d) $2^{\text {nd }}$ window NANF ${ }^{13}$ vs $1^{\text {st }}$ window NANF ${ }^{14}$ (SEM comparison in (e)), and simulated loss of two idealized first window NANFs.

To demonstrate the wide bandwidth transmission potential of this NANF, 2 similar spans were spliced into a single $~ 1 \mathrm{~km}$ long span, and high speed OOK/PAM4 data was transmitted through it across different telecom bands. In the experiments, two tunable lasers, covering the 1291-1372nm and 1461-1631nm wavelength ranges, were used to drive a Mach-Zehnder $\mathrm{LiNbO}_{3}$ modulator (MZM). The excellent modal purity of the fiber allowed penalty-free transmission of 100-Gbit/s PAM4 signals from the $\mathrm{O}$ to $\mathrm{L}-\mathrm{band}{ }^{14}$.

\section{THE FIRST HOLLOW CORE FIBRE WITH $<1 \mathrm{~dB} / \mathrm{KM}$ LOSS}

Theoretical studies of HCFs with a NANF structure have identified a wide range of geometric parameters which can, in principle, achieve losses comparable or even lower than $\mathrm{SMF}^{8,11,17,18}$. However, realizing such structures is a different challenge. In our most recent work, we reported that a low loss NANF operating in the first window can be realized with improvements in pressurization control, tube handling and preform assembly. With these improvements we were able to produce a fiber with a loss of $0.65 \mathrm{~dB} / \mathrm{km}$ across a bandwidth of $120 \mathrm{~nm}$, the first hollow core fiber to cross the $1 \mathrm{~dB} / \mathrm{km}$ threshold.

The fabricated NANF has a six nested tube design and a core size of $37.2 \mu \mathrm{m}$. All the regularly arranged tubes have the targeted thickness of $\sim 0.5 \mu \mathrm{m}$, which centers both the $\mathrm{C}$ and $\mathrm{L}$ bands in the low loss region of the first antiresonant window. Figure 1(d) shows a scanning electron micrograph (SEM) of the fiber cross section. The fiber has length of $1.23 \mathrm{~km}$, improving on the previous $0.5 \mathrm{~km}$ fiber ${ }^{13}$ and on the $0.62 \mathrm{~km}_{\text {fiber }}{ }^{14}$.

As can be seen in Fig. 1(d), the cross section of the fabricated fiber is considerably more symmetric than the previous attempt Fig. 1(e), with regularly spaced capillaries of similar diameters. The azimuthal gaps between the outer tubes, which control the amount of light leakage from the core, range from 3 to $5.8 \mu \mathrm{m}$ (average $4.6 \mu \mathrm{m}$ or $12 \%$ of the core diameter, and a standard deviation of $1.2 \mu \mathrm{m}$ ). One of the key features leading to the excellent optical performance of the fiber is the 
very good uniformity in membrane thickness. The 6 outer tubes have an average thickness of $0.49 \mu \mathrm{m}$, with a standard deviation of only $12 \mathrm{~nm}$. Crucially, the 6 inner tubes have a well-matched average thickness of $0.49 \mu \mathrm{m}$, with a standard deviation of $27 \mathrm{~nm}$. The regularity of this structure is well maintained along the $1.23 \mathrm{~km}$ length, with the other end showing a very similar geometry, and only a $2.7 \%$ smaller core size, illustrating the high level of structural control that can be achieved. Moreover, the OTDR trace shows no defects or scattering points along the full length.

Fig. 5(a) shows the broadband transmission through the full fiber spooled on a $1 \mathrm{~m}$ circumference bobbin, and its cut-back to $10 \mathrm{~m}$. A tungsten bulb white light source was injected into the fiber through butt coupling with an endlessly single mode photonic crystal fiber having a mode field diameter of $\sim 21 \mu \mathrm{m}$. The displayed spectra are the average of three repeated cleaves for both the short and long lengths. The corresponding measured average loss is shown in the solid red curve, with the lowest loss range form $1400-1700 \mathrm{~nm}$ expanded in Fig. 5(c), where the measurement uncertainty is also highlighted (greyed area around the curve). From $1520 \mathrm{~nm}$ to $1640 \mathrm{~nm}$ the fiber has an extremely flat attenuation of $\sim 0.65 \mathrm{~dB} / \mathrm{km}$ (with an absolute minimum measured loss of $0.63 \mathrm{~dB} / \mathrm{km}$ at $1553 \mathrm{~nm}$ ). The measurement uncertainties are estimated to be around $\pm 0.08 \mathrm{~dB} / \mathrm{km}$. The slightly higher loss between 1340 and $1460 \mathrm{~nm}$ is due to absorptions from the ingress of atmospheric water vapor into the fiber - no specific measures were taken to avoid atmospheric ingress throughout the measurement acquisition.
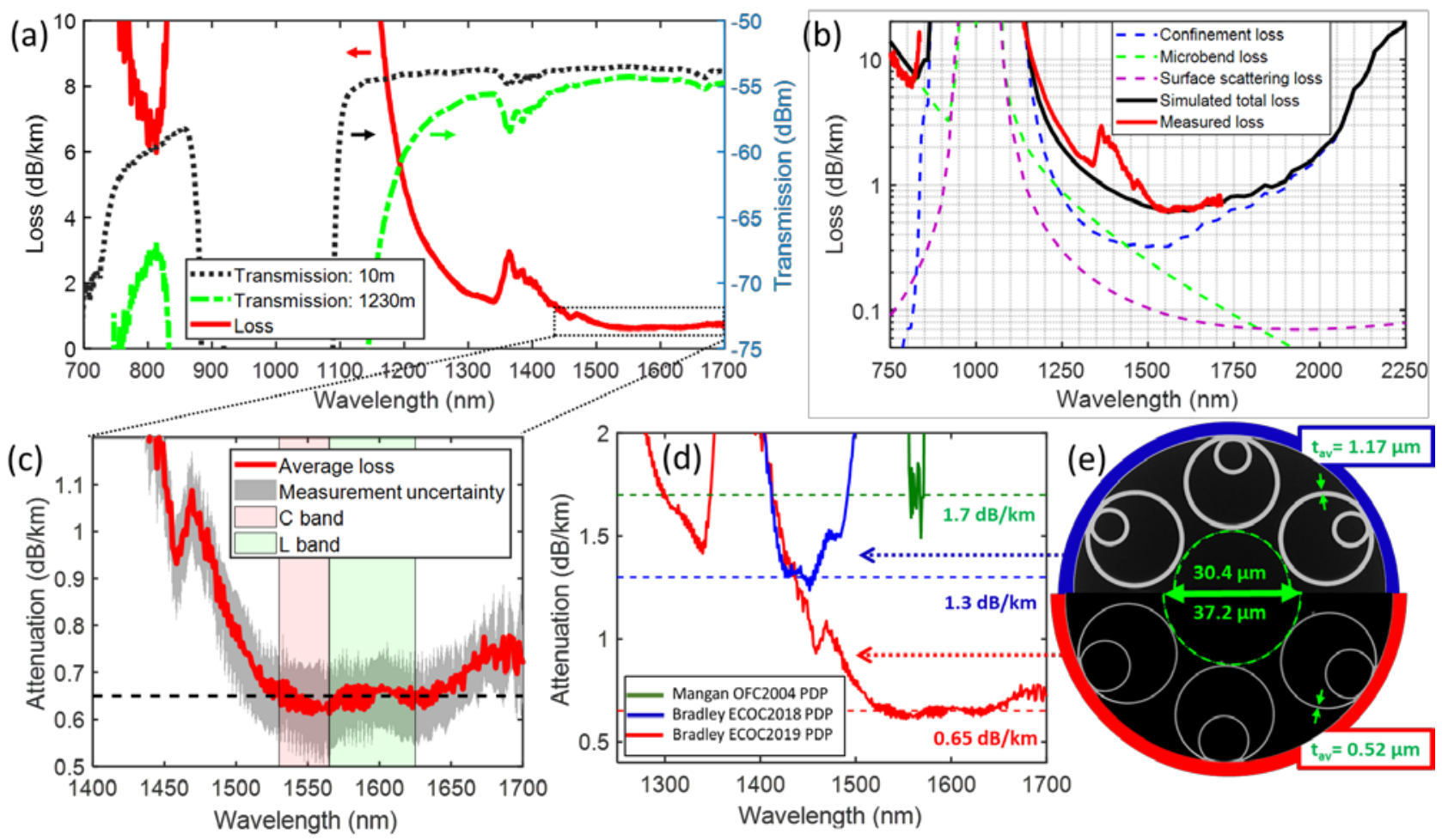

Figure 5: (a) Long (dashed green) and short (dotted black) length cut-back transmission spectra. Average cutback loss of the fiber (solid red); (b) Simulated vs measured attenuation of NANF and simulated individual loss contributions; (c) Magnified minimum attenuation region around $1550 \mathrm{~nm}$. The dashed line is at $0.65 \mathrm{~dB} / \mathrm{km}$; (d) Current fiber loss compared with that of previous low loss record HCFs; (e) Upper half: SEM of second window NANF ${ }^{13}$ and Lower half: SEM of first window NANF with loss $<1 \mathrm{~dB} / \mathrm{km}^{15}$.

In Fig. 5(b), the measured results are compared to finite element simulation performed using the commercial COMSOL multi-physics package. The simulation uses the NANF geometry extracted from Fig. 5(e) and mass conservation from the original tubes to accurately account for the membrane thicknes ${ }^{19}$. We can see that the measured and simulated fibre losses are in very good agreement (red and black solid curves respectively) in the first window, as well as in the second window, around $800 \mathrm{~nm}$. The simulations allow us to split the total loss into its confinement (dashed blue), micro bend (dashed green) and surface scattering (dashed purple) contributions. At $1550 \mathrm{~nm}$ the loss contributions due to leakage and microbending are $0.31 \mathrm{~dB} / \mathrm{km}$ and $0.24 \mathrm{~dB} / \mathrm{km}$, respectively. 
While these are already impressive results and very significant improvements over previous HCF state-of-the-art, we believe that it should be possible to further reduce these values with improvement in structure and coating design, respectively. Crucially, only $0.1 \mathrm{~dB} / \mathrm{km}$ is attributed to surface scattering, which is the only truly fundamental loss mechanism of the fibre. This provides assurance that further significant loss reduction is possible. Simulations also indicate that, as intended by design, a similar amount of leakage $(\sim 0.03 \mathrm{~dB} / \mathrm{km})$ occurs through each gap as occurs across each small tube, signifying a good level of structural optimisation.

In order to visualise the potential of this technology, we conduct a few simulations to understand the impact of future realistic improvements to the fibre. Figure 6 shows the experimental loss plot (red), compared with simulation performed on the reconstructed experimental geometry (black). With coating improvements to reduce microbend a $30 \%$ loss improvement at $1550 \mathrm{~nm}$ is predicted. Improving the symmetry made a further improvement of the order of $10 \%$ or so, since the experimental fibre was already fairly symmetric. Reducing the inter-tube azimuthal gap size to $3 \mu \mathrm{m}$ would have a very significant impact, halving the loss again to $\sim 0.2 \mathrm{~dB} / \mathrm{km}$, a loss not too dissimilar from that of conventional all-glass SMFs, throughout a large flat transmission window. Further improvement is still possible beyond that: increasing the core size by just $7 \%$, up to $40 \mu \mathrm{m}$, would half the loss yet again to a value below what is fundamentally achievable with conventional all-glass technology.

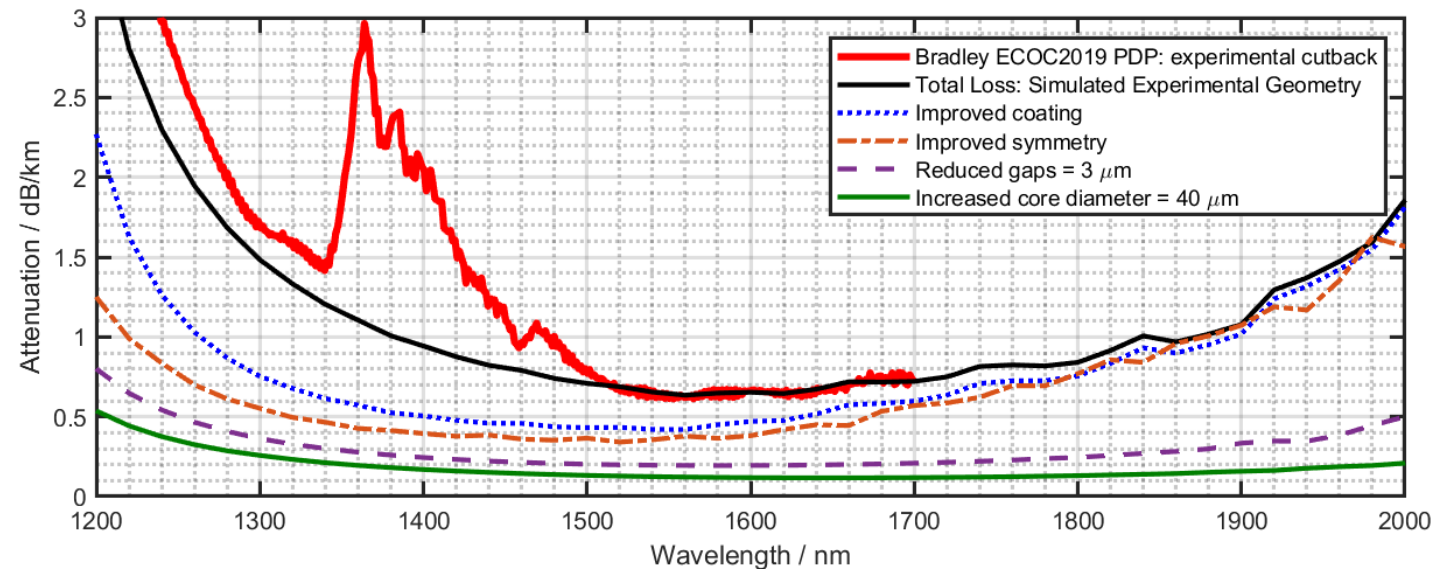

Figure 6. Simulations show a route to even lower loss, the total simulated loss using reconstructed geometry (black) agrees well with the experimental cutback ${ }^{15}$ (red). Further curves show reduced loss of accumulated improvements to coating, geometric symmetry, reduction in gap size between capillaries, and an increase in the core diameter from 37 to $40 \mu \mathrm{m}$.

\section{SUMMARY}

We have presented an overview of our latest results over the last 18 months in the development of effectively single mode, data-transmission-capable, low loss hollow core fibres. We have shown that NANFs can outperform all other known types of hollow core fiber in terms of loss, bandwidth and overall modal quality. Whilst impressive for a hollow core fiber, the current lowest loss value of $0.65 \mathrm{~dB} / \mathrm{km}$ has very considerable margin for improvements.

\section{ACKNOWLEDGEMENTS}

The authors would like to thank: Lucy Hooper, Marcelo Alonso, Arsalan Saljoghei and Mike Fake for their assistance; and we gratefully acknowledge the support of the Royal Academy of Engineering, and ERC (grant agreement no. 682724).

\section{REFERENCES}

[1] Cregan, R. F., Mangan, B. J., Knight, J. C., Birks, T. A., Russell, P. S., Roberts, P. J., and Allan, D. C., "Singlemode photonic band gap guidance of light in air," Science 285 (5433), 1537-1539 (1999). 
[2] Poletti, F., Wheeler, N. V., Petrovich, M. N., Baddela, N. K., Numkam Fokoua, E., Hayes, J. R., Gray, D. R., Li, Z., Slavik, R., and Richardson, D. J., "Towards high-capacity fibre-optic communications at the speed of light in vacuum," Nat. Photonics 7 (4), 279-284 (2013).

[3] Russell, P. S. J., Hölzer, P., Chang, W., Abdolvand, A., and Travers, J., "Hollow-core photonic crystal fibres for gas-based nonlinear optics," Nat. Photonics 8 (4), 278-286 (2014).

[4] Mangan, B., Farr, L., Langford, A., Roberts, P. J., Williams, D. P., Couny, F., Lawman, M., Mason, M., Coupland, S., and Flea, R., "Low loss $(1.7 \mathrm{db} / \mathrm{km})$ hollow core photonic bandgap fiber," Proc. Optical Fiber Communication Conference, PD24 (2004).

[5] Kolyadin, A. N., Kosolapov, A. F., Pryamikov, A. D., Biriukov, A. S., Plotnichenko, V. G., and Dianov, E. M., "Light transmission in negative curvature hollow core fiber in extremely high material loss region," Opt. Express 21 (8), 9514-9519 (2013).

[6] Debord, B., Amsanpally, A., Chafer, M., Baz, A., Maurel, M., Blondy, J., Hugonnot, E., Scol, F., Vincetti, L., and Gérôme, F., "Ultralow transmission loss in inhibited-coupling guiding hollow fibers," Optica 4 (2), 209-217 (2017).

[7] Hayes, J. R., Sandoghchi, S. R., Bradley, T. D., Liu, Z., Slavík, R., Gouveia, M. A., Wheeler, N. V., Jasion, G., Chen, Y., and Fokoua, E. N., "Antiresonant hollow core fiber with an octave spanning bandwidth for short haul data communications," J. Lightwave Technol. 35 (3), 437-442 (2017).

[8] Poletti, F., "Nested antiresonant nodeless hollow core fiber," Opt. Express 22 (20), 23807-23828 (2014).

[9] Jasion, G. T., Hayes, J. R., Wheeler, N. V., Chen, Y., Bradley, T. D., Richardson, D. J., and Poletti, F., "Fabrication of tubular anti-resonant hollow core fibers: Modelling, draw dynamics and process optimization," Opt. Express 27 (15), 20567-20582 (2019).

[10] Kosolapov, A. F., Alagashev, G. K., Kolyadin, A. N., Pryamikov, A. D., Biryukov, A. S., Bufetov, I. A., and Dianov, E. M., "Hollow-core revolver fibre with a double-capillary reflective cladding," Quantum Electronics 46 (3), 267 (2016).

[11] Antonio-Lopez, J. E., Habib, S., Van Newkirk, A., Lopez-Galmiche, G., Eznaveh, Z. S., Alvarado-Zacarias, J. C., Bang, O., Bache, M., Schülzgen, A., and Correa, R. A., "Antiresonant hollow core fiber with seven nested capillaries," Proc. IEEE Photonics Conference (IPC), 402-403 (2016).

[12] Gao, S. F., Wang, Y. Y., Ding, W., Jiang, D. L., Gu, S., Zhang, X., and Wang, P., "Hollow-core conjoined-tube negative-curvature fibre with ultralow loss," Nature communications 9 (1), 2828 (2018).

[13] Bradley, T. D., Hayes, J. R., Chen, Y., Jasion, G. T., Sandoghchi, S. R., Slavik, R., Fokoua, E. N., Bawn, S., Sakr, H., Davidson, I. A., Taranta, A., Thomas, J. P., Petrovich, M. N., Richardson, D. J., and Poletti, F., "Record low-loss 1.3db/km data transmitting antiresonant hollow core fibre," Proc. European Conference on Optical Communication (ECOC), 1-3, TH3F2 (2018).

[14] Sakr, H., Bradley, T. D., Hong, Y., Jasion, G. T., Hayes, J. R., Kim, H., Davidson, I. A., Fokoua, E. N., Chen, Y., Bottrill, K. R. H., Taengnoi, N., Petropoulos, P., Richardson, D. J., and Poletti, F., "Ultrawide bandwidth hollow core fiber for interband short reach data transmission," Proc. Optical Fiber Communication Conference (OFC), PD Th4A.1 (2019).

[15] Bradley, T., Jasion, G., Hayes, J., Chen, Y., Hooper, L., Sakr, H., Alonso, M., Taranta, A., Saljoghei, A., and Mulvad, H. C., "Antiresonant hollow core fibre with $0.65 \mathrm{db} / \mathrm{km}$ attenuation across the c and $\mathrm{l}$ telecommunication bands," Proc. European Conference on Optical Communication (ECOC), 1-3, PDP3.1 (2019).

[16] Fokoua, E. N., Chen, Y., Richardson, D. J., and Poletti, F., "Microbending effects in hollow-core photonic bandgap fibers," Proc. European Conference on Optical Communication (ECOC), 1-3 (2016).

[17] Belardi, W., and Knight, J. C., "Hollow antiresonant fibers with reduced attenuation," Opt. Lett. 39 (7), 18531856 (2014).

[18] Jasion, G. T., Richardson, D. J., and Poletti, F., "Novel antiresonant hollow core fiber design with ultralow leakage loss using transverse power flow analysis," Proc. Optical Fiber Communication Conference (OFC), 1-3, Th3E.2 (2019).

[19] Fokoua, E. N., Sandoghchi, S. R., Chen, Y., Jasion, G. T., Wheeler, N. V., Baddela, N. K., Hayes, J. R., Petrovich, M. N., Richardson, D. J., and Poletti, F., "Accurate modelling of fabricated hollow-core photonic bandgap fibers," Opt. Express 23 (18), 23117-23132 (2015). 